\title{
ALGEBRAIC ALGEBRAS WITH INVOLUTION
}

\author{
SUSAN MONTGOMERY
}

\begin{abstract}
The following theorem is proved: Let $R$ be an algebra with involution over an uncountable field $F$. Then if the symmetric elements of $R$ are algebraic, $R$ is algebraic.
\end{abstract}

In this paper we consider the following question:

"Let $R$ be an algebra with involution over a field $F$, and assume that the symmetric elements $S$ of $R$ are algebraic over $F$. Is $R$ algebraic over $F$ ?"

Previous results related to this question have been obtained by restricting the kind of algebraic relationships satisfied by the symmetric elements. For example, it was shown by Baxter and Martindale [1] for fields of characteristic not 2, and later by the author [5] for arbitrary fields, that if the symmetric elements are algebraic of bounded degree (or more generally, satisfy a polynomial identity), then $R$ must be algebraic. Another such result concerns rings whose symmetric elements are periodic (that is, for each $s \in S$, there is some integer $n(s)>1$ such that $s^{n(s)}=s$ ). In this case, the author has shown [6], [7] that $R$ must be algebraic; in fact $R$ satisfies a polynomial identity. When $R$ is a division ring, much more can be said: I. N. Herstein and the author [2] have shown that $R$ must actually be commutative. Finally, it has been shown by Osborn [8] that if $S$ is nil and $F$ is uncountable, then $R$ is nil. This answers for uncountable fields a question of McCrimmon [4, p. 391]:

"If $S$ is nil, is $R$ nil?"

An affirmative answer to this question in general would follow from an affirmative answer to the first question. For, as has been observed by both McCrimmon [4, p. 390] and Osborn [8, p. 306], if $S$ is nil then $R$ must be a radical ring. But if $R$ is algebraic, every element of the radical is nil; thus $R$ would be nil.

The result presented here differs from those described above in that no additional restrictions are imposed on the symmetric elements. We prove:

THEOREM. Let $R$ be an algebra with involution over an uncountable field $F$. Then if the symmetric elements of $R$ are algebraic, $R$ is algebraic.

Received by the editors January 8, 1971 .

AMS 1969 subject classifications. Primary 1658.

Key words and phrases. Rings with involution, algebraic algebras.

(c) American Mathematical Society 1972 
If $R$ is a ring, an involution on $R$ is simply an anti-automorphism of period 2. By an algebra with involution, we mean that $R$ has an involution * as a ring, and that the field $F$ has an automorphism $\alpha \rightarrow \bar{\alpha}$ of period 2 such that $(\alpha r)^{*}=\bar{\alpha} r^{*}$, for all $\alpha \in F, r \in R . S=\left\{x \in R \mid x^{*}=x\right\}$ will denote the symmetric elements of $R$.

Lemma 1. Let $R$ be an algebra with unit over a field $F$, and say $x \in R$ with $x^{2}=r x+s, r, s \in R$. Let $A=\left(\begin{array}{c}01 \\ r\end{array}\right)$, the $2 \times 2$ matrix. Then if $A$ is algebraic over $F, x$ is algebraic over $F$.

Proof. We first notice that $A^{2}=r A+s I$, where $I=\left(\begin{array}{ll}1 & 0 \\ 0 & 1\end{array}\right)$. Thus

$$
A^{3}=(r A+s I) A=r(r A+s I)+s A=\left(r^{2}+s\right) A+r s I=r_{1} A+s_{1} I .
$$

Similarly, $A^{n}=r_{n-2} A+s_{n-2} I$, where $r_{n-2}, s_{n-2} \in R$ for all $n>2$. Since $x$ satisfies $x^{2}=r x+s$, by the same procedure as for $A$ we find that also $x^{n}=r_{n-2} x+s_{n-2}$, for all $n>2$. Now if $A$ is algebraic over $F$, there exists some polynomial $p(\lambda) \in F[\lambda]$ such that $p(A)=0$. We claim that $p(x)=0$.

For, if $p(\lambda)=\lambda^{n}+\alpha_{n-1} \lambda^{n-1}+\cdots+\alpha_{1} \lambda+\alpha_{0}, \alpha_{i} \in F$, then

But

$$
\begin{aligned}
0= & p(A) \\
= & \left(r_{n-2} A+s_{n-2} I\right)+\alpha_{n-1}\left(r_{n-3} A+s_{n-3} I\right) \\
& +\cdots+\alpha_{2}(r A+s I)+\alpha_{1} A+\alpha_{0} I \\
= & \left(r_{n-2}+\alpha_{n-1} r_{n-3}+\cdots+\alpha_{2} r+\alpha_{1} 1\right) A \\
& +\left(s_{n-2}+\alpha_{n-1} s_{n-3}+\cdots+\alpha_{2} s+\alpha_{0} 1\right) I \quad \text { where } t, t^{\prime} \in R . \\
= & t A+t^{\prime} I, \quad
\end{aligned}
$$

$$
t A+t^{\prime} I=\left(\begin{array}{cc}
0 & t \\
t s & t r
\end{array}\right)+\left(\begin{array}{cc}
t^{\prime} & 0 \\
0 & t^{\prime}
\end{array}\right)=\left(\begin{array}{cc}
t^{\prime} & t \\
t s & t^{\prime}+t r
\end{array}\right),
$$

so $t A+t^{\prime} I=0$ implies $t=0$ and $t^{\prime}=0$. Since $x^{i}=r_{i-2} x+s_{i-2}, i>2, p(x)=$ $t x+t^{\prime}=0$ and thus $x$ is algebraic.

Recall that if $R$ is any algebra with unit, we may consider $R$ as an algebra of linear transformations by letting $R$ act on itself by right multiplication. Thus a characteristic root (or vector) of an element $r \in R$ will mean a characteristic root (or vector) of $r$ considered as a linear transformation acting by right multiplication. For any $r \in R$, we also define the spectrum $\sigma(r)=\{\alpha \in F$ such that $r-\alpha \cdot 1$ has no inverse in $R\}$. The resolvent $\rho(r)$ is the complement of $\sigma(r)$ in $F$.

LEMMA 2. Let $R$ be an algebra with involution over any field $F$ such that $S$ is algebraic. Assume that $R$ has a unit element, and that $F$ is fixed elementwise by *. Choose $x \in R$, and let $A=\left(\begin{array}{c}01 \\ s\end{array}\right)$, where $r=x+x^{*}$ and $s=-x^{*} x$. 
Consider $r$ acting by right multiplication on $R$, and $A$ acting by right multiplication on the $2 \times 2$ matrices over $R$. Then for any $\alpha \in \rho(r)$, the resolvent of $r$, with $\alpha \neq 0$, either $\alpha$ is a characteristic root of $A$ or $\alpha \in \rho(A)$, the resolvent of $A$.

Proof. Let $a=\left(\begin{array}{c}0 \\ 0\end{array}\right)$ and $y=\left(\begin{array}{c}0 \\ s\end{array}\right)$, so $A=a+y$. Let $\alpha \in F, \alpha \neq 0$. Assume that $a-\alpha I$ is invertible in $R_{2}$. Then there is some matrix $\left(\begin{array}{c}b c \\ d e\end{array}\right), b, c, d, e \in R$, such that $(a-\alpha I)\left(\begin{array}{ll}b & c \\ d & e\end{array}\right)=I$. Since $a-\alpha I=\left(\begin{array}{cc}-\alpha & 1 \\ 0 & r-\alpha\end{array}\right)$, this gives

$$
\left(\begin{array}{cc}
-\alpha & 1 \\
0 & r-\alpha
\end{array}\right)\left(\begin{array}{ll}
b & c \\
d & e
\end{array}\right)=\left(\begin{array}{cc}
-\alpha b+d & -\alpha c+e \\
(r-\alpha) d & (r-\alpha) e
\end{array}\right)=\left(\begin{array}{ll}
1 & 0 \\
0 & 1
\end{array}\right) .
$$

In particular, $(r-\alpha) e=1$ and so $(r-\alpha)^{-1}$ exists in $R$. Also $d=0, b=-1 / \alpha$, and $c=(r-\alpha)^{-1}$, and thus

$$
(a-\alpha I)^{-1}=\left(\begin{array}{cc}
-\frac{1}{\alpha} & \frac{1}{\alpha}(r-\alpha)^{-1} \\
0 & (r-\alpha)^{-1}
\end{array}\right) .
$$

Certainly if $\alpha \neq 0$ and $(r-\alpha)^{-1}$ exists in $R$, we have that $(a-\alpha I)^{-1}$ exists in $R_{2}$ by the expression for $(a-\alpha I)^{-1}$.

To summarize: if $\alpha \neq 0, a-\alpha I$ is invertible if and only if $r-\alpha$ is invertible. This means that $\rho(a) \subseteq \rho(r)$; in fact if $0 \notin \rho(r), \rho(a)=\rho(r)$, and if $0 \in \rho(r)$, $\rho(r)=\rho(a) \cup\{0\}$.

Choose $\alpha \in \rho(r), \alpha \neq 0$. Consider $A-\alpha I=(a-\alpha I)+y$. Multiplying on the right by $(a-\alpha I)^{-1}$ gives $I+y(a-\alpha I)^{-1}=I+y^{\prime}$, where $y^{\prime}=y(a-\alpha I)^{-1}$. We claim that $y^{\prime}$ is algebraic. For,

$$
y^{\prime}=\left(\begin{array}{ll}
0 & 0 \\
s & 0
\end{array}\right)\left(\begin{array}{cc}
-\frac{1}{\alpha} & \frac{1}{\alpha}(r-\alpha)^{-1} \\
0 & (r-\alpha)^{-1}
\end{array}\right)=\left(\begin{array}{cc}
0 & 0 \\
-\frac{s}{\alpha} & \frac{s}{\alpha}(r-\alpha)^{-1}
\end{array}\right) .
$$

Now $(s / \alpha)(r-\alpha)^{-1}=-\left(x^{*} x / \alpha\right)(r-\alpha)^{-1}$. Since $r-\alpha \in S,(r-\alpha)^{-1} \in S$ and thus $x\left(-(r-\alpha)^{-1} / \alpha\right) x^{*} \in S$. But then $x\left(-(r-\alpha)^{-1} / \alpha\right) x^{*}$ is algebraic, and so $x^{*} x\left(-(r-\alpha)^{-1} / \alpha\right)=(s / \alpha)(r-\alpha)^{-1}$ is algebraic. Say $t\left((s / \alpha)(r-\alpha)^{-1}\right)=0$, some polynomial $t(\lambda)$. We may assume that $t(\lambda)$ has no constant term (multiply by $\lambda$ if necessary). Thus $t\left(y^{\prime}\right) \in\left(\begin{array}{l}0 \\ R\end{array}\right)$, so $t\left(y^{\prime}\right)^{2}=0$, and $y^{\prime}$ is algebraic.

For an algebraic element, the spectrum coincides with the characteristic roots of the linear transformation [3, p. 246]. Hence either $-1 \in \rho\left(y^{\prime}\right)$ or -1 is a characteristic root of $y^{\prime}$.

If $-1 \in \rho\left(y^{\prime}\right)$, then $\left(I+y^{\prime}\right)^{-1}$ exists, and so

$$
((a+y)-\alpha)^{-1}=(a-\alpha I)^{-1}\left(I+y^{\prime}\right)^{-1}
$$


and $\alpha \in \rho(A)$. But if -1 is a characteristic root of $y^{\prime}$, then there is an $x \neq 0$, $x \in R_{2}$, so that $x\left(I+y^{\prime}\right)=0$. Then $x(a+y-\alpha I)=x\left(I+y^{\prime}\right)(a-\alpha I)=0$, and $\alpha$ is a characteristic root of $A$.

Proof of the Theorem. Choose $x \in R$. Then $x^{2}-\left(x+x^{*}\right) x+x^{*} x=0$; letting $r=x+x^{*}$ and $s=-x^{*} x$, we have $x^{2}=r x+s$. Thus by Lemma 1 , it is enough to show that $A=\left(\begin{array}{ll}0 & 1 \\ s & r\end{array}\right)$ is algebraic.

First note that we may assume that $F$ is left elementwise fixed by the automorphism - . For if not, let $F_{0}$ be the subfield of $F$ fixed elementwise by.$- R$ is certainly an algebra over $F_{0}, F_{0}$ is uncountable, and $F$ is algebraic over $F_{0}$ (as " - " has period 2). Thus if $s \in S$ is algebraic over $F, s$ is algebraic over $F_{0}$. Thus $R$ satisfies the hypotheses as an algebra over $F_{0}$. But if $R$ is algebraic over $F_{0}, R$ is certainly algebraic over $F$.

We may also assume that $R$ contains a unit element. For if not, consider the algebra $R_{1}=\{(r, \alpha) \mid r \in R, \alpha \in F\}$, where addition is defined componentwise and multiplication is given by $(r, \alpha) \cdot(t, \beta)=(r t+\alpha t+\beta r, \alpha \beta)$. $R_{1}$ has an involution, given by $(r, \alpha)^{*}=\left(r^{*}, \alpha\right)$, and is an algebra over $F$ by $\alpha(r, \beta)=(\alpha r, \alpha \beta)$. Now the symmetric elements of $R_{1}$ are algebraic: let $(s, \alpha)$ be a symmetric element. Since $s \in S, s$ is algebraic over $F$, say $p(s)=0$, where $p(\lambda) \in F[\lambda]$. Then $(s, \alpha)$ satisfies the polynomial $p(\lambda-\alpha)$, so is algebraic. Certainly if $R_{1}$ is algebraic, $R$ is algebraic.

Finally, we may assume that $R$ is finitely generated over $F$-if not, replace $R$ by $R^{\prime}=F\left[1, x, x^{*}\right]$. This means that the dimension of $R$ over $F$ is countable.

We apply Lemma 2 to see that for any $\alpha \in \rho(r)$, either $\alpha$ is a characteristic root of $A$ or $\alpha \in \rho(A)$. But $\rho(r)$ is uncountable, since the spectrum of $r$ consists of the roots of its minimal polynomial [3, p. 20], and so either $\rho(A)$ is uncountable or the set of distinct characteristic roots of $A$ is uncountable. The latter is impossible, for then $R_{2}$, the $2 \times 2$ matrices, would contain an uncountable number of characteristic vectors, which are linearly independent. This contradicts the dimension of $R_{2}$ over $F$ being countable.

Thus it must be that $\rho(A)$ is uncountable, and so $A$ is algebraic [3, p. 20].

AdDED IN PROOF. Kevin McCrimmon now has a more direct proof of the theorem.

\section{REFERENCES}

1. W. E. Baxter and W. S. Martindale III, Rings with involution and polynomial identities, Canad. J. Math. 20 (1968), 465-473. MR 36 \#5168.

2. I. N. Herstein and S. Montgomery, A note on division rings with involution, Michigan Math. J. 18 (1971), 75-79.

3. N. Jacobson, Structure of rings, 2nd rev. ed., Amer. Math. Soc. Colloq. Publ., vol. 37, Amer. Math. Soc., Providence, R.I., 1964. MR 36 \#5158.

4. K. McCrimmon, On Herstein's theorems relating Jordan and associative algebras, J. Algebra 13 (1969), 382-392. MR 40 \#2721. 
5. S. Montgomery, Polynomial identity algebras with involution, Proc. Amer. Math. Soc. 27 (1971), 53-56.

6. - A generalization of a theorem of Jacobson, Proc. Amer. Math. Soc. 28 (1971), 366-370.

7. - A generalization of a theorem of Jacobson. II (to appear).

8. J. M. Osborn, Jordan and associative rings with nilpotent and invertible elements, J. Algebra 15 (1970), 301-308.

Department of Mathematics, University of Southern California, Los Angeles, California 90007 